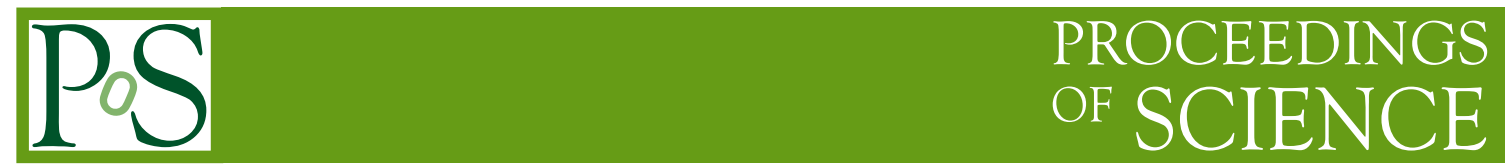

\title{
TOP counter for Belle II - post installation R\&Ds
}

\author{
Raita Omori *, Genta Muroyama *, Noritsugu Tsuzuki *, for the Belle II TOP Group \\ Nagoya University \\ E-mail: raitadhepl.phys.nagova-u.ac.jp \\ murovamaehepl.phys.nagova-u.ac.jp, \\ noritsugudhepl.phys.nagoya-u.ac.jp
}

We developed a new particle identification device, named TOP counter, for the next B-factory "Belle II" experiment. We produced 17 TOP counter modules and installed 16 modules into the Belle II structure.

We will present the R\&D items after the installation, such as issues on operation in the magnetic field, further investigation to achieve a longer lifetime of the MCP-PMT and the performance of the readout electronics.

The 3rd International Symposium on "Quest for the Origin of Particles and the Universe" 5-7 January 2017

Nagoya University, Japan

\footnotetext{
* Speaker
} 


\section{Introduction}

The TOP counter [U] is a ring imaging Cherenkov detector for the $\pi^{ \pm} / K^{ \pm}$particle identification (PID) in the Belle II experiment []. //We have finished TOP installation into the Belle II detector in May, 2016.// The TOP counter mainly consists of a quartz bar, photon sensors and readout electronics. Cherenkov photons are generated and propagate in the quartz bar. The TOP counter uses the difference of the time of propagation for PID. In the case of $3 \mathrm{GeV} / \mathrm{c} \pi^{ \pm}$and $K^{ \pm}$, the time difference is about $100 \mathrm{ps}$. Therefore, the required time difference is $50 \mathrm{ps}$. It is satisfied by using the MCP-PMT [ 团] and the waveform sampling ASIC [3]. In addition, because the number of detected photon is about 20, a high photon detection efficency is essential.

Fig.W shows the structure of the TOP counter electronics and the structure of the PMT module. Readout electronics are connected to PMT module. The MCP-PMTs are installed into the TOP counter as a set of modules with 4 PMTs. They are glued to the wave length cut filter and the front borad by silicon glue.
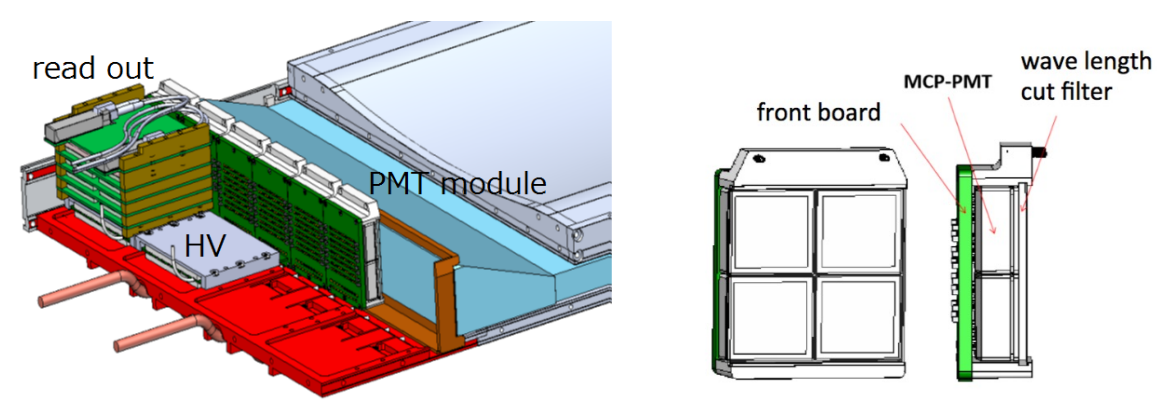

Figure 1: The structure of the TOP module around the PMT module (left) and the PMT module structure (right).

The performance of the installed modules were tested in $1.5 \mathrm{~T}$ of the Belle II solenoid. We checked the photon detection efficiency using laser light irradiating the MCP-PMTs in situ. We found that some PMT modules are rotated by the magnetic force o the MCP-PMTs and some photons were lost by the air bubbles which appeared at the optical contact between the quartz and the wavelength cut filter. The issue is discussed in section 2 .

Another concern is drop of photon efficiency by deterioration of MCP-PMT photocathode during the long-period Belle II run. We have studied for the improvement of the MCP-PMT lifetime, which is described in section 3.

We need a time calibration for the ASICs to have a good time resolution. We measured each sampling time gap because they are not uniform sample by sample. The detail is written in section 4.

\section{Measurements for the PMT rotation problem}

Because the tube of the MCP-PMT is made of magnetic material, the MCP-PMTs are affected by the magnetic force from 1.5 T magnetic field of the Belle II solenoid. Some PMTs rotate like a compass in the PMT module and their window peeled off from wavelength cut filter. This is a 
problem because such PMTs decline photon efficiency. The cause is not only that the PMT receives $1 \mathrm{kgf}$ from $1.5 \mathrm{~T}$ magnetic force, but also that there is a space between the PMT and the front board.

A possible solution of this problem is inserting a flat shim into the gap between the PMT and the front board (D). To confirm usefulness of putting shim, we performed load tests to measure how much force makes the PMT window peel off from the wavelength cut filter. In the test, the PMT is fixed by a vise and a spring applies force to only the module flame (Fig.[B]).

We prepared three types of shim with the thickness of $0.5,1.5$ and $1.6 \mathrm{~mm}$. The $0.5 \mathrm{~mm}$ shim is used for tuning the difference of the PMT thickness. We found that the shim could prevent the PMT from detaching off the filter against force of $3-4 \mathrm{kgf}$ in this test. This result shows that the PMTs with the shim will not rotate in $1.5 \mathrm{~T}$. Further studies on a long term stability are now on going.
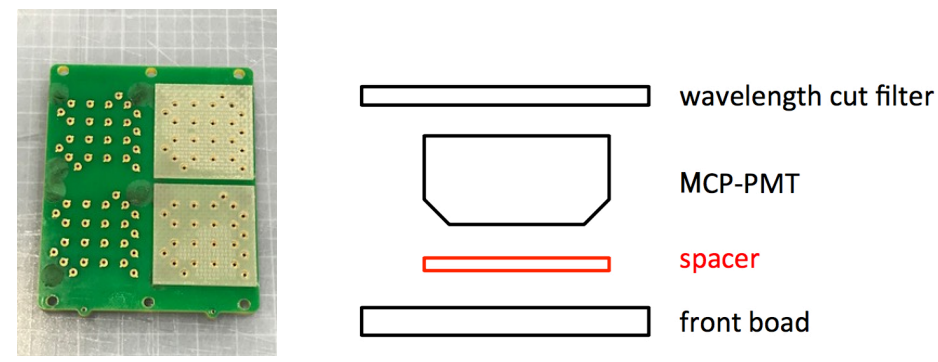

Figure 2: The shim on the front board to fill the space between the MCP-PMT and the front board. White boads in left picture are shims. Right figure is schematic side view of the PMT module with shim.

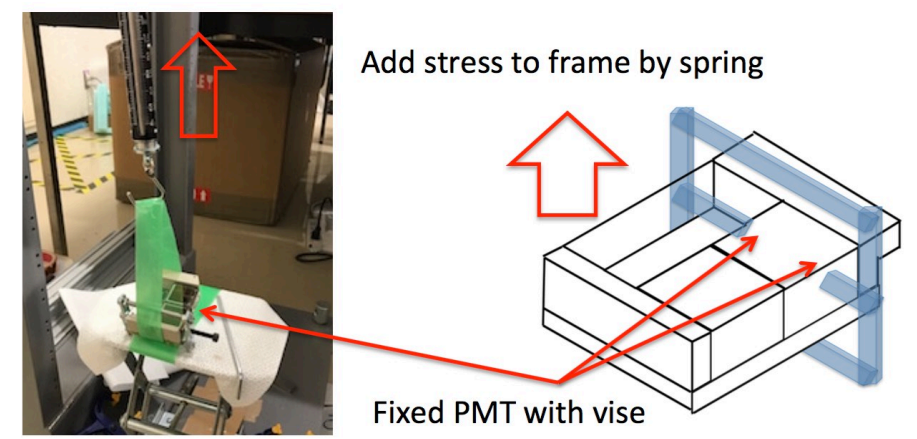

Figure 3: The load test setting. The PMT is fixing and a spring applies force to only the PMT module flame. A blue flame in right figure means the vise.

\section{Measurement of after pulse for improvement of the MCP-PMT lifetime}

The lifetime of the MCP-PMT photocathode is an important issue to operate the MCP-PMT under a high background rate. We have studied this issue and produced three types of MCP-PMTs, called conventional, ALD and Life-extended ALD MCP-PMT [5]]. The lifetime is, respectively, estimated to be about one year and eight years on average and at least 15 years under the current background estimation in the Belle II experiment.

One of the sources of the photocathode deterioration is known to be ion feedback from the MCP to the photocathode [目]. Therefore, we measured the feedback ion rate using after pulses 
initiated when the feedback ions hit the photocathode. The delay time of the after pulse from the main pulse is a function of the mass of the ion : $t \propto \sqrt{m_{\text {ion }}}$. Using this relation we are able to identify the ion species. We measured the after pulse rate for the three types of MCP-PMTs by using two CAMAC TDCs. The pico pulse laser is used as a light source. The MCP-PMT signal is divided into two. One is fed to the one of the TDCs to measure the main pulse timing. Another is fed to the other TDC followed by a veto of the main pulse.
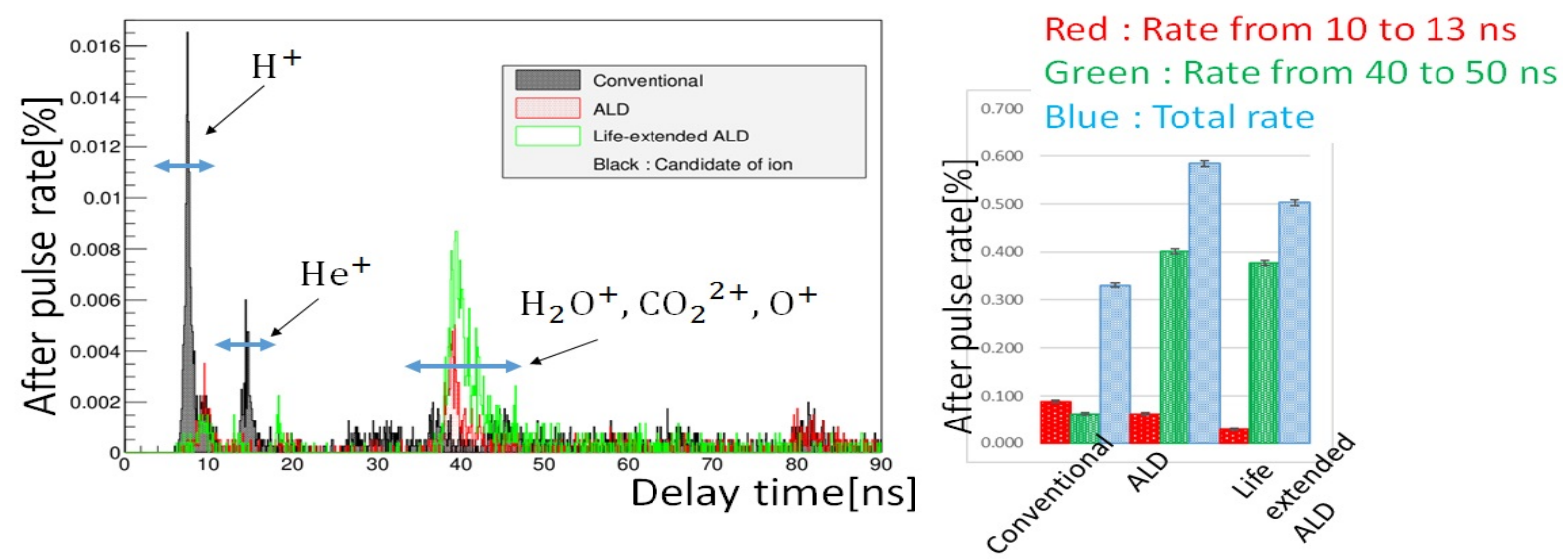

Figure 4: Distribution of the delay time of the after pulse from the main pulse (left) and the average of the after pulse rate (right) for each type of the MCP-PMT.

The result is shown in Fig.t. Left histogram is the distribution of the delay time of the after pulse from the main pulse. From the applied voltage and the gap length between the photocathode and the MCP, we consider the first peak around $10 \mathrm{~ns}$ as $\mathrm{H}^{+}$originated from the MCP and the second peak around $15 \mathrm{~ns}$ as $\mathrm{He}^{+}$. The third peak around $40 \mathrm{~ns}$ corresponds to the mass number from 16 to 28 if it is a single charged ion. The right graph is the average of the after pulse rate for the three types of MCP-PMTs. It shows that the ion contributions are different for each type. The conventional MCP-PMTs have a larger amount of $\mathrm{H}^{+}$and $\mathrm{He}^{+}$than the ALD and life-extended ALD MCP-PMTs. A different ion is found in the these two ALD coating types. Candidates are $\mathrm{H}_{2} \mathrm{O}^{+}, \mathrm{CO}_{2}^{2+}$ or $\mathrm{O}^{+}$. We also found that the total after pulse rate has no correlation with lifetime of the MCP-PMTs. We will check a correlation of the lifetime with the gain of the MCP-PMT.

\section{Sampling time calibration (measurement) on TOP electronics}

The readout ASICs sample waveforms of the MCP-PMTs at a $2.7 \mathrm{GHz}$ sampling rate. Each ASIC channel has a window of 256 samplings and repeats it while data taking. The time range of window is fixed, but each sampling interval is not uniform. To get a 50 ps time resolution of the readout electronics, we need to calibrate the sampling intervals.

Fig. $[1$ shows the method to calculate the 256 sampling intervals of an ASIC. We input two calibration pulses with a constant time difference $(=\mathrm{T})$. The number of samples between the two pulses varies depending on the first pulse timing, and we can get 256 expressions (the sum of the sampling intervals between the two pulses is equal to T). We calculate the 256 sample intervals using these expressions. 
The time resolution of the readout electronics improves from hundreds ps to $<50 \mathrm{ps}$ after the calibration. It meets the requirement of the TOP counter.
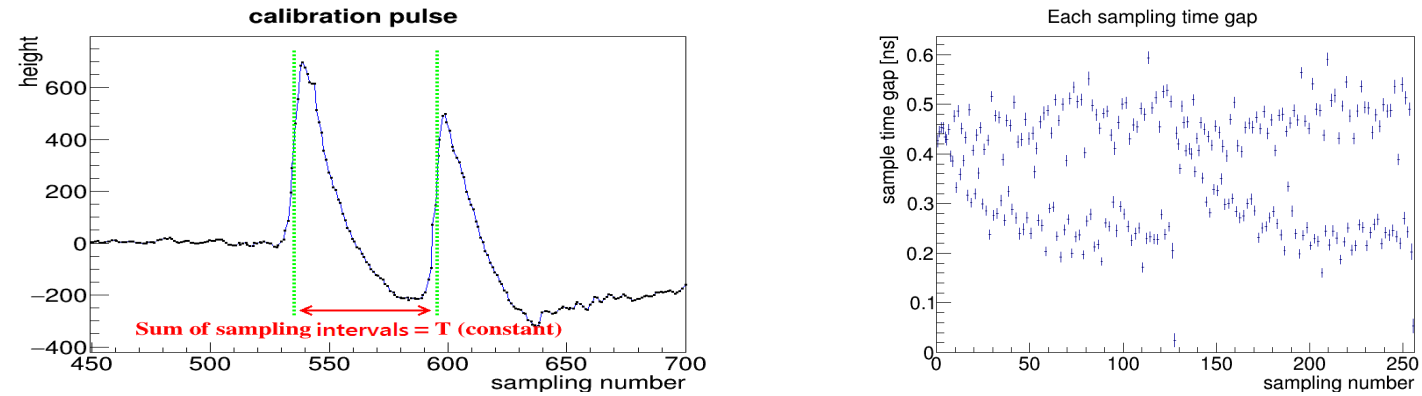

Figure 5: Two input calibration pulses with a constant time difference T read by the ASIC (left). Calculated sampling interval at each sampling (right).

\section{Summary}

The installation of the TOP counter was finished and we are preparing for the Belle II physics run. The MCP-PMT rotation in the PMT module could be resolved by putting a shim in the gap between the MCP-PMT and the front board. We are now testing it in a long term. We will fix the all PMTs in the TOP counter before Belle II physics run on late 2018. We could see the effect of ion feedback by the after pulse and found no correlation between the total after pulse rate and the MCP-PMT lifetime. We will check a relationship among the ion feedback, the MCP-PMT lifetime and the applied voltage to improve the lifetime. The calibration of the sampling intervals in the ASICs works well. The time resolution including the MCP-PMT will be checked by using the laser.

\section{Acknowledgments}

We are grateful to the Belle II TOP group members for helpful discussions on our research. This work was supported by MEXT Grant-in-Aid for Scientific Research on Innovative Areas "Elucidation of New Hadrons with a Variety of Flavors" and Grant-in-Aid for Scientific Research (S) "Probing New Physics with Tau-Lepton" (No. 26220706).

\section{References}

[1] M. Akatsu et al., Nucl. Instr. and Meth. A440 (2000) 124 ; T. Ohshima, ICFA Instr. Bull. 20 (2000) 10 ; T. Ohshima,.Nucl. Instr. and Meth. A453 (2000) 331

[2] T.Abe et al., KEK-REPORTS-2010-1, 2010.

[3] Matthew Andrew, PoS TIPP2014 (2014) 171

[4] M. Akatsu et al., Nucl. Instr. and Meth. A528 (2004) 763 ; K. Inami et al., Nucl. Instr. and Meth. A560 (2006) 303 ; K. Inami et al., Nucl. Instr. and Meth. A592 (2008) 247

[5] K. Matsuoka, et al., Nucl. Instr. and Meth. A, in press, http://dx.doi.org/10.1016/j.nima.2017.02.010.

[6] N. Kishimoto et al., Nucl. Instr. and Meth. A564 (2006) 204 ;T.Jinno et al., Nucl. Instr. and Meth. A629 (2011) 111 